\title{
Utilidad del uso o no de isquemia en el postquirúrgico inmediato en la artroplastia total de rodilla
}

\author{
Usefulness of the use or not of ischemia in the immediate \\ postoperative period in total knee arthroplasty \\ Ángel López Valencia, * Rafael Bustos Mora ${ }^{\ddagger}$

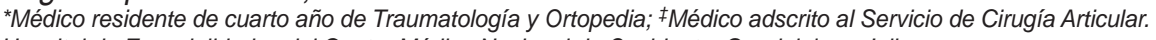 \\ Hospital de Especialidades del Centro Médico Nacional de Occidente. Guadalajara, Jalisco.
}

\section{Resumen}

La artroplastia total de rodilla se considera el principal tratamiento de la artrosis de rodilla. El uso de la isquemia con asa neumática o elástica con ventajas técnico-quirúrgicas como la mejora de la visión quirúrgica al mantener un campo operatorio más limpio y la reducción del tiempo operatorio, no es un procedimiento seguro. Objetivo: Comparar la eficacia y seguridad de la artroplastia total de rodilla con o sin uso de isquemia en el Centro Médico Nacional de Occidente «Lic. Ignacio García Téllez». Material y métodos: Se trata de un estudio observacional, transversal, retrospectivo y monocéntrico. Pacientes con evaluación ambulatoria del Servicio de Ortopedia y Traumatología con diagnóstico de artrosis de rodilla que requirieron manejo quirúrgico con artroplastia total de rodilla primaria y con componentes de revisión, con y sin uso de isquemia donde se evaluó el tiempo de cirugía, la hemorragia, el dolor, la necesidad de transfusión y la diferencia de hemoglobina a las 24 horas del postoperatorio de marzo de 2017 a marzo de 2020. Resultados: Se analizaron 75 pacientes, la edad media fue de 71 años, con una desviación estándar de 8.2, 46 pacientes sin uso de isquemia y 29 con uso de isquemia. En los pacientes en los que se utilizó la isquemia, se observó una escala visual analógica (EVA) superior a 5 en $51.7 \%$, un Andersen superior a 2 en $51.7 \%$, el tiempo de cirugía fue inferior o igual a 150 minutos en $93.1 \%$, la hemorragia postoperatoria fue inferior o igual a $500 \mathrm{ml}$ en $93.1 \%$, no fue necesaria ninguna transfusión de sangre en $96.6 \%$ y una diferencia de hemoglobina ( $\mathrm{g} / \mathrm{dll}$ ) de 2 o superior en $82.8 \%$. En los pacientes que no utilizaron isquemia, se observó una EVA de 5 o menos en $91.3 \%$, un Andersen de 2 o menos en $91.3 \%$, el tiempo de la cirugía fue mayor de 150 minutos en $69.6 \%$, la hemorragia postoperatoria fue menor o igual a $500 \mathrm{ml}$ en $60.9 \%$, se requirió transfusión de sangre en $19.6 \%$ y se encontró una diferencia de hemoglobina $(\mathrm{g} / \mathrm{dl})$ de 2 o más en $89.1 \%$. Conclusiones: Los pacientes que utilizan la isquemia en la artroplastia total de rodilla tienen menos sangrado, tiempo quirúrgico y necesidad de transfusión de sangre, pero su uso aumenta el dolor postoperatorio.

Palabras clave: Artroplastia total de rodilla, uso de isquemia, dolor, tiempo de cirugía, hemoglobina.

\begin{abstract}
The total knee arthroplasty is considered the main treatment for knee osteoarthritis. The use of ischemia with a pneumatic or elastic handle with technical-surgical advantages, such as an improvement in surgical vision by maintaining a cleaner operative field and reducing operative time, is not a safe procedure. Objective: To compare the efficacy and safety of total knee arthroplasty with or without use of ischemia in the Centro Médico Nacional de Occidente «Lic. Ignacio García Téllez». Material and methods: This is an observational, cross-sectional, retrospective and single-center study. From March 2017 to March 2020, patients with an outpatient evaluation of the Orthopedics and Traumatology Service with a diagnosis of knee osteoarthritis who required surgical management with primary total knee arthroplasty and with revision components, with and without the use of ischemia where The surgery time, bleeding, pain, the need for transfusion and the difference in hemoglobin at 24 hours postoperative were assessed. Results: 75 patients were analyzed, the average age was 71 years, with a standard deviation of 8.2, 46 patients without use of ischemia and 29 with use of ischemia. In patients in whom ischemia was used, a VAS greater than 5 was observed in $51.7 \%$, an Andersen greater than 2 in $51.7 \%$, the surgery time was less than or equal to 150 minutes in $93.1 \%$, bleeding postoperative was less than or equal to $500 \mathrm{ml}$ in $93.1 \%$, no blood transfusion was required in $96.6 \%$ and a hemoglobin difference ( $\mathrm{g} / \mathrm{dl}$ ) of 2 or greater in $82.8 \%$. In patients who did not use ischemia, a VAS 5 or less was observed in $91.3 \%$, an Andersen of 2 or less in 42 in $91.3 \%$, the time of surgery was greater than 150 minutes in $69.6 \%$, postoperative bleeding
\end{abstract}

Correspondencia:

Dr. Ángel López Valencia

E-mail: angel_lo_4@hotmail.com

Recibido: 17-05-2021. Aceptado: 12-07-2021.

Citar como: López VÁ, Bustos MR. Utilidad del uso o no de isquemia en el postquirúrgico inmediato en la artroplastia total de rodilla. Orthotips. 2021; 17 (4): 190-194. https://dx.doi.org/10.35366/102215 
was less than or equal to $500 \mathrm{ml}$ in $60.9 \%$, blood transfusion was required in $19.6 \%$ and a hemoglobin difference (g/dl) of 2 or greater was found in $89.1 \%$. Conclusion: The patients who use ischemia in total knee arthroplasty have less bleeding, surgical time and need for blood transfusion, but its use increases postoperative pain.

Keywords: Total knee arthroplasty, use of ischemia, pain, surgery time, hemoglobin.

\section{Introducción}

La osteoartrosis es el trastorno musculoesquelético más común encontrado en atención primaria. ${ }^{1}$ Como una enfermedad crónica está caracterizada por dolor y discapacidad física, siendo la rodilla la articulación más afectada. ${ }^{2}$ Según la OMS, afecta a 9.6\% de los hombres y $18 \%$ de las mujeres $>60$ años. ${ }^{3}$ En EUA, la tasa de incidencia anual estandarizada para la gonartrosis por edad y sexo por 100,000 habitantes fue de $240 .{ }^{4}$ En la mayoría de los casos, vamos a encontrar que no existe ningún factor determinante que origine la aparición de artrosis sin existir ninguna alteración articular preexistente, dentro de las causas secundarias destacan las postquirúrgicas, postinfecciosas y postraumáticas, donde desempeñan un papel fundamental las deformidades angulares residuales como el varo y valgo, que provocan distribución anormal de las cargas de peso. ${ }^{5}$ La presencia de factores de riesgo contribuye al aumento de la incidencia de la enfermedad en la población general, y se correlaciona con el grado de dolor articular experimentado por el paciente. ${ }^{6}$ Se ha encontrado relación de la obesidad y el tejido adiposo, ya que aumenta la síntesis de citocinas proinflamatorias, como el factor de necrosis tumoral alfa y las interleucinas (IL) 1, 6, 8 y 18, disminuyendo además las citocinas reguladoras como la $\mathrm{IL}-10 .{ }^{7}$ Se considera el dolor como síntoma primario y la principal causa de discapacidad funcional. ${ }^{8}$ Para la gradación radiológica de la gonartrosis se ha desarrollado la escala de Ahlbäck (Tablas 1 y 2) y la de Kellgren y Lawrence (Figura 1).9-11 La artroplastia total de rodilla (Figura 2) es considerada como el principal tratamiento y constituye al día de hoy el que consigue mejores resultados funcionales y de satisfacción a corto y mediano plazo. ${ }^{12}$ El uso de isquemia con mango neumático o elástico sigue muy arraigado en la práctica de la cirugía ortopédica, si bien es cierto que conlleva ventajas técnico-quirúrgicas, como una mejora en la visión quirúrgica al mantener un campo operatorio más limpio y reduce el tiempo operatorio, no es un procedimiento inocuo, ${ }^{13}$ ya que se le ha asociado con complicaciones trombóticas, vasculares, nerviosas, dolor intenso en el muslo, retardo en la cicatrización de la herida, cambios hemodinámicos inherentes al proceso de isquemia y reperfusión y disminución de rangos de movilidad. ${ }^{14,15}$

\section{Material y métodos}

Se trata de un estudio de tipo observacional, transversal, retrospectivo y unicéntrico. Durante el periodo de marzo de 2017 a marzo de 2020, se realizó una investigación y revisión de expedientes clínicos de la Unidad Médica de Alta Especialidad (UMAE) del Centro Médico Nacional de Occidente «Lic. Ignacio García Téllez» en el Servicio de Traumatología y Ortopedia y de nuestro sistema digital
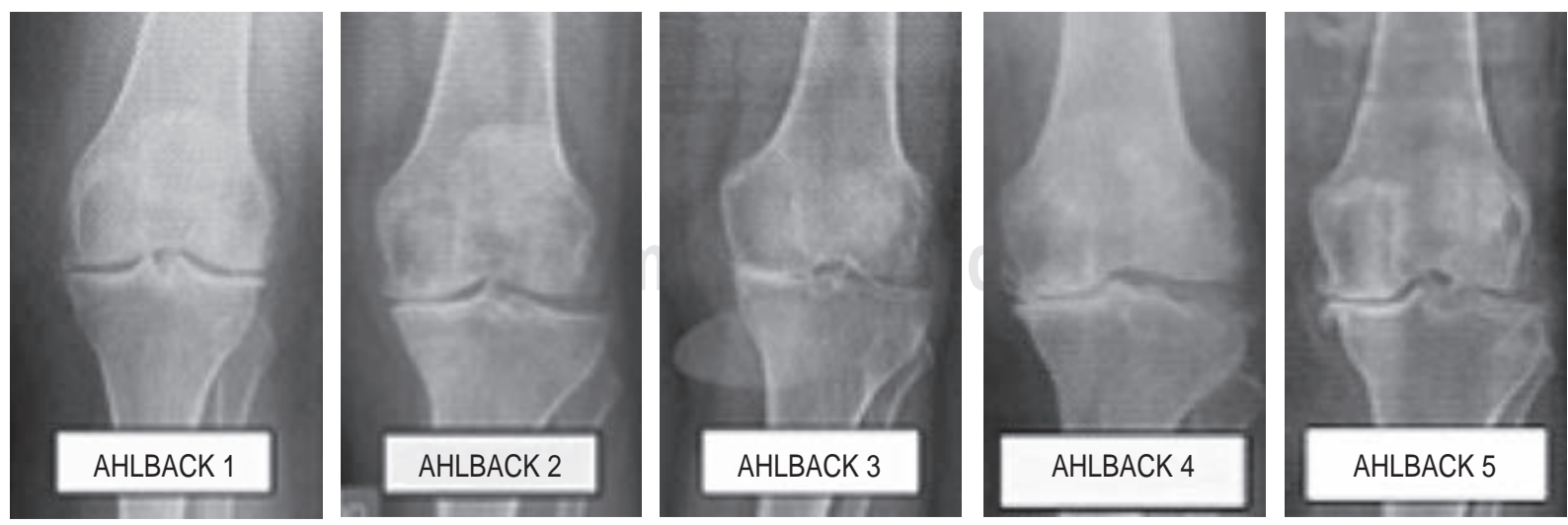

Figura 1: Clasificación de Ahlbäck. 
Tabla 1: Clasificación de Ahlbäck.

\begin{tabular}{cl}
\hline Grado & Definición \\
\hline I & Disminución de la interlínea en $50 \%$ \\
III & Desaparición de la interlínea \\
IV & Erosión ósea leve $<0.5 \mathrm{~cm}$ \\
V & Erosión ósea moderada de 0.5 a $1 \mathrm{~cm}$ \\
\hline
\end{tabular}

Tabla 2: Clasificación radiológica de osteoartrosis según Kellgren y Lawrence.

\begin{tabular}{ll}
\hline Grado & Característica radiográfica \\
\hline 0 & Normal \\
1 (OA dudosa) & $\begin{array}{l}\text { Dudoso estrechamiento del espacio articular. } \\
\text { Posibles osteofitos }\end{array}$ \\
2 (OA leve) & $\begin{array}{l}\text { Posible estrechamiento del espacio articular. } \\
\text { Osteofitos }\end{array}$ \\
3 (OA moderada) & $\begin{array}{l}\text { Estrechamiento del espacio articular. Osteofitos } \\
\text { moderados múltiples. Leve esclerosis. Posible } \\
\text { deformidad de los extremos de los huesos }\end{array}$ \\
4 (OA grave) & $\begin{array}{l}\text { Marcado estrechamiento del espacio articular. } \\
\text { Abundantes osteofitos, Esclerosis grave, } \\
\text { deformidad de los extremos de los huesos }\end{array}$ \\
\hline
\end{tabular}

Evernote. Se realizó la captación de los datos clínicos del sistema digital, donde se encuentran radiografías anteroposteriores (AP) de rodilla y lateral con flexión de $30^{\circ}$ y mediante la escala de Kellgren-Lawrence se identificó el grado de artrosis. Se realizó la revisión de expedientes clínicos para la obtención de los siguientes datos: laboratorios prequirúrgicos, el uso o no de isquemia transquirúrgica, tiempo de duración de cirugía, tiempo de duración de isquemia, sangrado operatorio mediante el conteo de gasas, compresas y contenedores de aspiración cotejado entre el equipo quirúrgico y el de anestesia, el dolor postoperatorio a las 24 horas será registrado mediante la escala visual análoga del dolor y escala de Andersen, laboratorios postquirúrgicos y necesidad de transfusión sanguínea, siendo ésta necesaria con hemoglobinas por debajo de $9.0 \mathrm{~g} / \mathrm{dl}$ acompañadas de sintomatología de bajo gasto, disnea, vértigo, náuseas, palidez tegumentaria y de mucosas. Se realizó análisis estadístico con la aplicación Epi Info con tabla $2 \times 2$.

Criterios de inclusión: paciente adulto (mayor de 18 años) y afiliado de forma vigente al IMSS, con diagnóstico de gonartrosis grado III y IV de acuerdo con la clasificación de Kellgren-Lawrence y que requirieron manejo quirúrgico mediante artroplastia total de rodilla con componentes primarios.

Criterios de no inclusión: pacientes con presencia de lesión o fractura en otra extremidad del cuerpo, con antecedente de cirugía previa en la rodilla, con presencia de neuropatía de miembros inferiores que limita la percepción del dolor previo a la lesión, con antecedente de enfermedad sanguínea o manejo con medicamentos anticoagulantes por patología previa, con catéter peridural para manejo del dolor postoperatorio o intolerancia a los antiinflamatorios no esteroideos (AINE) y lesiones en la piel antes de realizar el evento quirúrgico en el sitio de abordaje.

\section{Resultados}

Se analizaron 75 pacientes, la edad promedio fue de 71 años, con una desviación estándar de 8.2, en un rango de 52 a 86 años, 45 pacientes $(60 \%)$ fueron hombres y $30(40 \%)$ mujeres, 46 pacientes $\sin$ uso de isquemia y 29 con uso de isquemia, $86.66 \%$ presentaban artrosis grado IV en la clasificación de Kellgren y Lawrence, 92\% presentaban deformidad en varo. El lado que se operó con mayor frecuencia fue el derecho con $61.33 \%$. En los pacientes en los que se utilizó isquemia se observó un EVA mayor de 5 en 15 pacientes (51.7\%), un Andersen mayor de 2

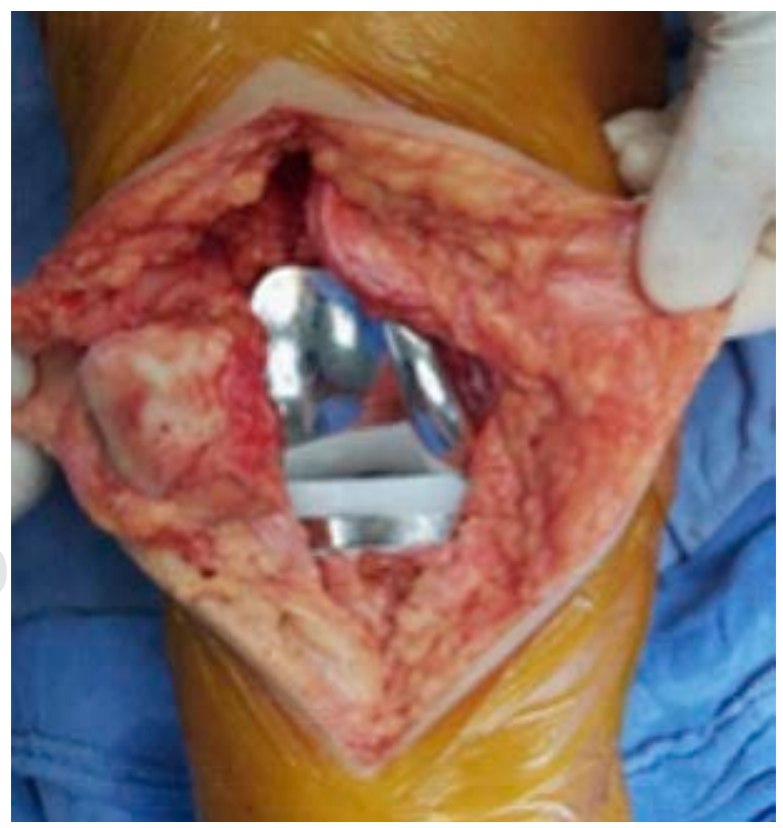

Figura 2: Prótesis total de rodilla primaria. 
en 15 (51.7\%), el tiempo de cirugía fue menor o igual a 150 minutos en 27 casos (93.1\%), el sangrado postquirúrgico fue menor o igual a $500 \mathrm{ml}$ en 27 (93.1\%), no se requirió transfusión sanguínea en 28 (96.6\%) y se encontró una diferencia de hemoglobina $(\mathrm{g} / \mathrm{dl}) \mathrm{de}$ 2 o mayor en 24 pacientes (82.8\%). En los casos que no se utilizó isquemia se observó un EVA 5 o menor en 42 pacientes (91.3\%), un Andersen de 2 o menor en $42(91.3 \%)$, el tiempo de cirugía fue mayor a 150 minutos en $32(69.6 \%)$, el sangrado postquirúrgico fue menor o igual a $500 \mathrm{ml}$ en $28(60.9 \%)$, se requirió transfusión sanguínea en nueve (19.6\%) y se encontró

Tabla 3: Resultados descriptivos.

Datos clínicos y epidemiológicos de pacientes con cirugía de prótesis de rodilla con y sin isquemia

\begin{tabular}{|c|c|c|}
\hline & $\begin{array}{c}\text { Con isquemia } \\
\mathrm{N}=29(38.7 \%) \\
n(\%)\end{array}$ & $\begin{array}{c}\text { Sin isquemia } \\
\mathrm{N}=46(61.3 \%) \\
\mathrm{n}(\%)\end{array}$ \\
\hline \multicolumn{3}{|l|}{ Edad (años) } \\
\hline Mayor de 60 & 24 (82.8) & $29(63.0)$ \\
\hline 60 o menor & $5(17.2)$ & $17(37.0)$ \\
\hline \multicolumn{3}{|l|}{ Género } \\
\hline Masculino & $19(65.5)$ & $26(56.5)$ \\
\hline Femenino & $10(34.5)$ & $20(43.5)$ \\
\hline \multicolumn{3}{|c|}{ Grado de artrosis (Kellgren y } \\
\hline \multicolumn{3}{|l|}{ Lawrence) } \\
\hline Ill o menor & $0(0)$ & $10(21.7)$ \\
\hline IV & $29(100.0)$ & $36(78.3)$ \\
\hline \multicolumn{3}{|l|}{ Deformidad } \\
\hline Varo & $29(100.0)$ & $40(87.0)$ \\
\hline Valgo & $0(0)$ & $6(13.0)$ \\
\hline \multicolumn{3}{|l|}{ Rodilla intervenida } \\
\hline Izquierda & $10(34.5)$ & $19(41.3)$ \\
\hline Derecha & $19(65.5)$ & $27(58.7)$ \\
\hline \multicolumn{3}{|c|}{ Dolor (escala visual analógica) } \\
\hline 5 o menor & $14(48.3)$ & $42(91.3)$ \\
\hline$>5$ & $15(51.7)$ & $4(8.7)$ \\
\hline \multicolumn{3}{|l|}{ Dolor (Andersen) } \\
\hline 20 menor & $14(48.3)$ & $42(91.3)$ \\
\hline$>2$ & $15(51.7)$ & $4(8.7)$ \\
\hline \multicolumn{3}{|c|}{ Tiempo de cirugía (min) } \\
\hline Mayor de 150 & $2(6.9)$ & $32(69.6)$ \\
\hline 150 o menor & $27(93.1)$ & $14(30.4)$ \\
\hline \multicolumn{3}{|l|}{ Sangrado (ml) } \\
\hline 500 o menor & $27(93.1)$ & $28(60.9)$ \\
\hline Mayor de 500 & $2(6.9)$ & $18(39.1)$ \\
\hline \multicolumn{3}{|c|}{ Necesidad de transfusión } \\
\hline Sí & $1(3.4)$ & $9(19.6)$ \\
\hline No & $28(96.6)$ & $37(80.4)$ \\
\hline \multicolumn{3}{|c|}{ Diferencia de hemoglobina ( $\mathrm{g} / \mathrm{dl})$} \\
\hline Menor de 2 & $5(17.2)$ & $5(10.9)$ \\
\hline 20 mayor & $24(82.8)$ & $41(89.1)$ \\
\hline
\end{tabular}

Tabla 4: Resultados analíticos.

\begin{tabular}{lccc}
\hline & OR & IC del 95\% & p \\
\hline $\begin{array}{l}\text { Dolor evaluado con } \\
\text { escala EVA }\end{array}$ & 0.0889 & $0.0253-0.3127$ & 0.00004791 \\
$\begin{array}{l}\text { Dolor evaluado con } \\
\text { escala Andersen }\end{array}$ & 0.0889 & $0.0253-0.3127$ & 0.00004791 \\
$\begin{array}{l}\text { Tiempo de cirugía } \\
\begin{array}{l}\text { Sangrado postquirúrgico } \\
\text { Necesidad de }\end{array}\end{array}$ & 0.0324 & $0.0068-0.1554$ & 0.00000004 \\
transfusión sanguínea & 0.1468 & $0.0176-1.276$ & 0.04346094 \\
$\begin{array}{l}\text { Diferencia de } \\
\text { hemoglobina }\end{array}$ & 1.7083 & $0.4482-6.5116$ & 0.32441268 \\
\hline
\end{tabular}

EVA = escala visual analógica; $\mathrm{OR}$ = razón de momios; $\mathrm{IC}$ = intervalo de confianza.

una diferencia de hemoglobina $(\mathrm{g} / \mathrm{dl})$ de 2 o mayor en 41 pacientes $(89.1 \%)$ (Tabla 3).

Los datos sobre los resultados analíticos se muestran en la Tabla 4.

\section{Discusión}

El uso de isquemia en la cirugía articular y en general en la cirugía ortopédica y traumatología es una práctica cotidiana. ${ }^{14}$ La utilidad de su uso es controlar el sangrado durante la cirugía. ${ }^{16}$ Nuestros resultados encontraron diferencia estadísticamente significativa $(p<0.05)$ en la relación entre el uso de isquemia y la disminución de sangrado, menor necesidad de transfusión y menor tiempo quirúrgico, y en los pacientes sin uso de isquemia en relación con menor dolor postquirúrgico en el Centro Médico Nacional de Occidente Guadalajara, Jalisco.

En 2009, Toby O Smith muestra una revisión sistemática que refiere que el uso de torniquete mejora la visualización operatoria. ${ }^{17}$ En el 2012, Trueba-Davalillo describe que la utilización de la isquemia en la artroplastia total de rodilla nos ayuda a disminuir el tiempo quirúrgico y la aplicación de transfusión, esto ayuda a tener una mejor recuperación y más rápida integración a las actividades diarias, en esta investigación corroboramos estos datos teniendo como resultado una menor pérdida sanguínea y menor tiempo quirúrgico tras el uso de isquemia. ${ }^{16}$

En 2015, Fang-Zhen Jiang realiza un metaanálisis que indica que el uso de isquemia podría reducir la pérdida de sangre intraoperatoria, la tasa de transfusión y tiempo de operación; sin embargo, se asocia con problemas menores como dolor, hinchazón y 
trombosis venosa, este fenómeno también se observó en este estudio, puesto que los pacientes que fueron expuestos a isquemia refieren mayor dolor postquirúrgico. ${ }^{18}$ En 2016, Gutiérrez-García JA mostró que el diferencial de hemoglobina y sangrado operatorio fue menor cuando se asoció al uso de isquemia, datos similares a nuestros resultados. ${ }^{14}$

La investigación tiene fortalezas en el apartado de la metodología, ya que se encuentra con una evaluación de variables cuantitativas en el momento quirúrgico, tales como sangrado y tiempo quirúrgico, las cuales se realizaron por todo el equipo quirúrgico. Dentro de las variables de diferencia de hemoglobina y la necesidad de transfusión sanguínea, se utilizaron exámenes sanguíneos prequirúrgicos y postquirúrgicos de nuestro hospital, que ofrecen una certeza y equidad en los resultados. Dentro de las debilidades se considera a la escala EVA y Andersen, que aunque es muy utilizada en el ámbito mundial, en varios estudios no son muy confiables ya que son subjetivas dependiendo del paciente. Otro punto a destacar sería valorar el consumo tanto de analgésicos como de opioides de rescate en el periodo postquirúrgico para determinar la eficacia analgésica.

\section{Conclusiones}

Se encontró mediante esta investigación que los pacientes en quienes se utiliza isquemia en la artroplastia total de rodilla presentan menor sangrado, tiempo quirúrgico y necesidad de transfusión sanguínea, pero su uso aumenta el dolor postquirúrgico. Se recomienda utilizar con cautela la isquemia y su uso queda a elección del cirujano, conociendo las ventajas de su uso a través del mejor control de sangrado y disminuyendo tiempos quirúrgicos para así evitar posibles complicaciones.

\section{Referencias}

1. Reginato AM, Riera $H$, Vera M, Torres AR, Espinosa $R$, Esquivel Valerio JA, et al. Osteoarthritis in Latin America study of demographic and clinical characteristics in 3040 patients. J Clin Rheumatol. 2015; 21 (8): 391-397.

2. Tratamiento con artroplastia de rodilla en pacientes mayores de 60 años. México: Secretaría de Salud; 2011.

3. Marquina J. Gonartrosis, presentación de un caso y revision de la literatura. España: Universidad de Valladolid; 2013.

4. Mena Pérez R. Caracterización de pacientes con gonartrosis de rodilla. Centro de Diagnóstico Integral "Concepción". Rev Haban Cienc Méd [Internet]. 2016; 15 (1): 17-26. Disponible en:
http://scielo.sld.cu/scielo.php?script=sci_arttext\&pid=S1729519X2016000100004\&lng=es

5. Álvarez-López CA, García-Lorenzo YC. Relación entre deformidad angular y gonartrosis primaria. Acta Ortop Mex. 2015; 29 (4): 232-236.

6. Borges PA, Almeida MHJ, Araújo LM, Fukuyama JM, Umada FY, Arab MGL. Correlation between Ahlbäck classification and gonarthrosis risk factors. Acta Ortop Bras. 2018; 26 (4): 240-243.

7. Charles-Lozoya S, Treviño-Báez JD, Ramos-Rivera JA, Rangel-Flores JM, Tamez-Montes JC, Brizuela-Ventura JM. Síndrome metabólico y otros factores asociados a gonartrosis. Gac Med Mex. 2017; 153 (7): 775-780.

8. Neogi T, Frey-Law L, Scholz J, Niu J, Arendt-Nielsen L, Woolf $\mathrm{C}$, et al. Sensitivity and sensitisation in relation to pain severity in knee osteoarthritis: trait or state? Ann Rheum Dis. 2015; 74 (4): 682-688.

9. Meza-Reyes G, Aldrete-Velasco J, Espinosa-Morales R, Torres-Roldán F, Díaz-Borjón A, Robles-San Román M. Osteoarthrosis: implementation of current diagnostic and therapeutic algorithms. Rev Med Inst Mex Seguro Soc [Internet]. 2017; 55 (1): 67-75. Available in: http://www.ncbi. nlm.nih.gov/pubmed/28092250

10. Nahum A. Book review. Am J Dig Dis. 1968; 13 (8): 766.

11. Martínez Figueroa R, Martínez Figueroa C, Calvo Rodríguez R, Figueroa Poblete D. Osteoartritis (artrosis) de rodilla. Rev Chil Ortop Traumatol. 2015; 56 (3): 45-51.

12. Kurtz S, Ong K, Lau E, Mowat F, Halpern M. Projections of primary and revision hip and knee arthroplasty in the United States from 2005 to 2030. J Bone Joint Surg Am. 2007; 89 (4): 780-785.

13. Olivecrona C, Lapidus LJ, Benson L, Blomfeldt R. Tourniquet time affects postoperative complications after knee arthroplasty. Int Orthop. 2013; 37 (5): 827-832.

14. Gutiérrez-García JA, Sierra-Pérez M, García-Velazco RA, Salas-Mora CA, Cisneros-González VM. Artroplastía total cementada de rodilla: comparación entre el uso o no de isquemia en el resultado postoperatorio inmediato. Acta Ortop Mex. 2016; 30 (1): 7-12.

15. Watanabe H, Kikkawa I, Madoiwa S, Sekiya H, Hayasaka S, Sakata Y. Changes in blood coagulation-fibrinolysis markers by pneumatic tourniquet during total knee joint arthroplasty with venous thromboembolism. J Arthroplasty [Internet]. 2014; 29 (3): 569-573. Available in: http://dx.doi.org/10.1016/j. arth.2013.08.011

16. Trueba-Davalillo C, Suárez-Ahedo CE, Trueba-Vasavilbaso C, Obil-Chavarría C, Gil-Orbezo F. Uso de isquemia en la artroplastía total de rodilla. ¿Existe diferencia? Acta Ortop Mex. 2012; 26 (2): 112-115.

17. Smith TO, Hing CB. A meta-analysis of tourniquet assisted arthroscopic knee surgery. Knee [Internet]. 2009; 16 (5): 317-321. Available in: http://dx.doi.org/10.1016/j. knee.2009.01.004

18. Jiang FZ, Zhong HM, Hong YC, Zhao GF. Use of a tourniquet in total knee arthroplasty: a systematic review and meta-analysis of randomized controlled trials. J Orthop Sci. 2015; 20 (1): 110-123.

\section{Conflicto de intereses}

Sin conflicto de intereses. 